\title{
Technical variations in prostatic immunohistochemistry: need for standardisation and stringent quality assurance in PSA and PSAP immunostaining
}

\author{
M Varma, D M Berney, B Jasani, A Rhodes
}

J Clin Pathol 2004;57:687-690. doi: 10.1136/icp.2003.014894

\begin{abstract}
Aims: To assess variations in prostate specific antigen (PSA) and prostate specific acid phosphatase (PSAP) immunohistochemistry with particular reference to the antibody type (monoclonal or polyclonal) and the tissues used for optimising immunostaining conditions and as external positive controls.

Methods: A questionnaire was sent to all laboratories registered with the UK National External Quality Assurance Scheme for immunohistochemistry enquiring about the immunohistochemical methods routinely used for the diagnosis of prostate cancer. Results: Responses were received from $220(68 \%)$ laboratories. All UK respondents routinely performed PSA immunostaining but PSAP immunostaining was available in only $57 \%$ of these laboratories. Monoclonal anti-PSA, polyclonal anti-PSA, monoclonal anti-PSAP, and polyclonal anti-PSAP were used by $40 \%, 60 \%, 29 \%$, and $27 \%$ of UK respondents, respectively. Benign prostate tissue was most commonly used to determine optimal antibody dilutions and as external quality control for PSA/PSAP, with only $6 \%$ and $3 \%$ of respondents, respectively, including high grade prostate cancer in the tissues used for these purposes.

Conclusions: The wide variation in the methods used highlights the need for standardisation and more stringent quality assurance of the immunohistochemical staining techniques used for PSA and PSAP. The widespread use of benign prostate tissue to determine optimal antibody dilutions and as an external positive control for PSA and PSAP immunostaining is of particular concern because this approach may result in a method that is not sufficiently sensitive to detect the reduced PSA and PSAP expression associated with high grade prostate cancer.
\end{abstract}

See end of article for authors' affiliations

Correspondence to: Dr M Varma, Department of Histopathology, University Hospital of Wales, Heath Park, Cardiff CF14 4XN, Wales, UK; Murali.Varma@ cardiffandvale.wales. nhs.uk

Accepted for publication 5 February 2004

$\mathrm{R}$ ecent editorials and review papers have highlighted the lack of standardisation of techniques used for diagnostic immunohistochemistry, resulting in difficulties when comparing findings from different laboratories, ${ }^{12}$ This situation appears to be true with the immunohistochemical diagnosis of prostate cancer.

Accurate identification of the prostatic origin of metastatic carcinoma is important because prostate cancer, even in advanced stages, may respond to hormonal manipulation. Serum prostate specific antigen (PSA) values may help to establish the prostatic origin of poorly differentiated carcinoma. However, some tumours, although expressing PSA immunohistochemically, may secrete relatively small amounts of the glycoprotein into the bloodstream. Conversely, urothelial carcinomas extending into the prostate gland are often associated with raised serum PSA. ${ }^{3}$ Hence, immunohistochemistry remains the definitive method for establishing the diagnosis in morphologically difficult cases. PSA and prostate specific acid phosphatase (PSAP) are two of the few tumour type specific immunohistochemical markers currently available. Both monoclonal and polyclonal antibodies are available for the immunohistochemical detection of PSA and PSAP. However, there is little information in the literature regarding the relative specificity and sensitivity and frequency of use of these antibodies, either singly or in combination, for establishing prostatic differentiation in the context of an unknown primary and with regard to the pretreatment methods used to improve the sensitivity of PSA and PSAP immunostaining in formalin fixed tissues.

Another source of concern in PSA and PSAP immunohistochemistry relates to the choice of tissues for quality control of immunostaining. PSA and PSAP are most often used to establish the prostatic origin of poorly differentiated carcinoma. It has been shown that poorly differentiated prostate cancer expresses significantly less PSA and PSAP than benign prostate or low grade prostate cancer. ${ }^{45}$ Hence, the use of these tissues for quality control of PSA and PSAP immunostaining of poorly differentiated prostate cancer may be inappropriate. We believe that many laboratories currently use benign prostate tissue to determine antibody dilutions and as an external positive control in PSA and PSAP immunohistochemistry, although data to substantiate this belief are not available.

"Poorly differentiated prostate cancer expresses significantly less prostate specific antigen and prostate specific acid phosphatase than benign prostate or low grade prostate cancer" $^{\prime \prime}$

This potential laboratory to laboratory variation in PSA and PSAP sensitivity could have serious clinical implications as encountered in a recent consultation case. A transurethral prostate resection showed poorly differentiated carcinoma that was negative on monoclonal anti-PSA immunostaining performed twice at another institution and reported as "consistent with transitional cell carcinoma". However, repeat immunostaining at our institution showed diffuse immunoreactivity with the more sensitive polyclonal antibody to PSA and patchy positivity for PSAP, indicating a diagnosis of

Abbreviations: PSA, prostate specific antigen; PSAP, prostate specific acid phosphatase; UKNEQAS-IHC, UK National External Quality Assurance Scheme for immunohistochemistry 


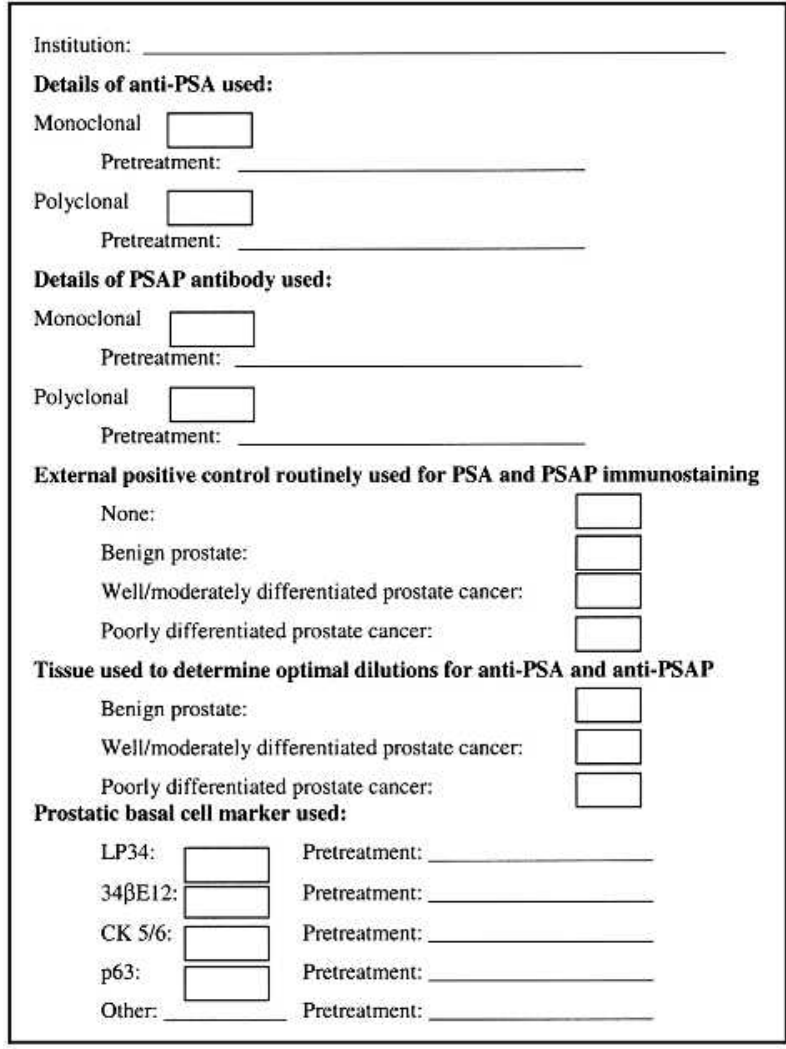

Figure 1 Questionnaire circulated to all subscribers to the United Kingdom National External Quality Assurance Scheme for immunohistochemistry. PSA, prostate specific antigen; PSAP, prostate specific acid phosphatase.

prostate cancer. Follow up revealed rising serum PSA and extensive skeletal metastasis in keeping with prostate cancer.

Therefore, we conducted a postal survey of immunohistochemical methods used in the diagnosis of prostate cancer in laboratories registered with the UK National External Quality Assurance Scheme for immunohistochemistry (UKNEQASIHC). The findings are presented to highlight the need for standardisation of the methods and to establish more stringent quality assurance.

\section{METHODS}

A short questionnaire was circulated to all laboratories subscribing to the UKNEQAS-IHC scheme in 2003 (fig 1). To maximise the response rate, the questionnaire along with a stamped and addressed envelope was circulated with the external quality assurance slides in two successive runs of the scheme. Duplicated responses were identified and excluded.

From the replies received, we determine the frequency of use of PSA and/or PSAP, the type of anti-PSA and anti-PSAP antibodies used (monoclonal or polyclonal), and the nature of the tissues used as external positive control and to optimise antibody dilutions.

We plan to present the survey findings related to basal cell markers in a separate report.

\section{RESULTS}

The questionnaire was circulated to 394 laboratories, 323 of which were known to subscribe to the prostate cancer immunohistochemistry external quality assurance exercise. Of these 323 laboratories, 196 were UK laboratories.

After excluding duplicates, 220 (68\%) replies were received; 133 from the UK and 87 from outside the UK ( 13 from the Republic of Ireland, 58 from continental Europe, and 16 from elsewhere). Therefore, the response rate for UK laboratories was $68 \%$ (133 of 196).

Because almost all UK laboratories practising diagnostic prostate immunohistochemistry are known to participate in the UKNEQAS-IHC scheme, the response received from them can be considered to be representative. The situation regarding UKNEQAS-IHC participation by laboratories outside the UK is not known so the responses from these nonUK laboratories may not be representative of the practice in these countries. Therefore, these data were considered separately from those from UK laboratories.

Table 1 summarises the responses regarding the choice of prostate marker (PSA and PSAP), primary antibody for each marker (monoclonal or polyclonal), and antigen "retrieval" methods used. All the laboratories that offered a routine service for prostate marker immunohistochemistry performed PSA immunostaining. However, PSAP immunostaining was made available as a routine marker in only $57 \%$ of such laboratories in the UK and 53\% elsewhere. Monoclonal and polyclonal antibodies were both frequently used by the respondents to demonstrate PSA and PSAP. In general, respondents used polyclonal antibodies to PSA and PSAP without pretreatment, whereas heat induced epitope retrieval (HIER) was the most frequently used pretreatment method for the monoclonal anti-PSA and anti-PSAP antibodies.

Table 2 describes the choices of tissue to determine optimal antibody dilution and as a routine external positive control for PSA/PSAP immunohistochemistry. The most commonly used tissue for both the above purposes was benign prostate, used either alone or in conjunction with well/moderately differentiated prostate cancer. Poorly differentiated prostate cancer, alone or with benign prostate or well to moderately differentiated prostate cancer was used to determine the optimal dilutions for the anti-PSA and anti-PSAP antibodies

Table 1 Details of PSA and PSAP immunostaining methods

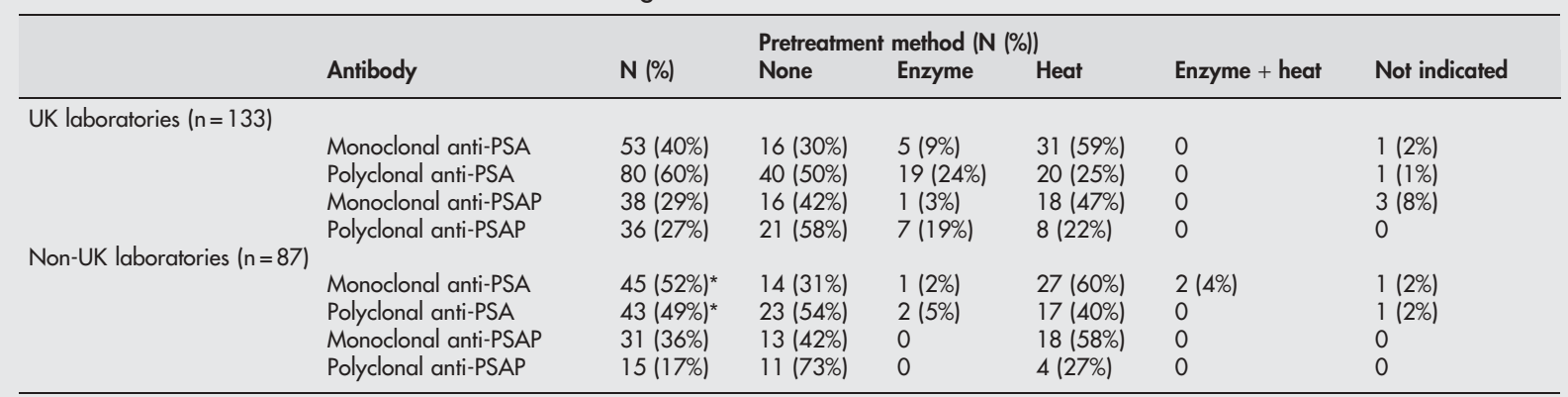

*One centre used both monoclonal and polyclonal antibodies to PSA.

PSA, prostate specific antigen; PSAP, prostate specific acid phosphatase. 
Table 2 Choice of tissue for quality control of PSA and PSAP immunohistochemistry

\begin{tabular}{|c|c|c|c|c|}
\hline \multirow[b]{2}{*}{ Tissue } & \multicolumn{2}{|c|}{ Optimisation of antibody dilutions } & \multicolumn{2}{|c|}{ External positive control } \\
\hline & $\begin{array}{l}\text { UK laboratories } \\
\mathrm{N}(\%)\end{array}$ & $\begin{array}{l}\text { Non-UK laboratories } \\
\mathrm{N}(\%)\end{array}$ & $\begin{array}{l}\text { UK laboratories } \\
\mathrm{N}(\%)\end{array}$ & $\begin{array}{l}\text { Non-UK laboratories } \\
\mathrm{N}(\%)\end{array}$ \\
\hline Not indicated & $11(8 \%)$ & $7(8 \%)$ & $5(4 \%)$ & $6(7 \%)$ \\
\hline None & 0 & $2(2 \%)$ & $11(8 \%)$ & $6(7 \%)$ \\
\hline Benign prostate & $84(63 \%)$ & $65(75 \%)$ & $82(62 \%)$ & $67(77 \%)$ \\
\hline Benign prostate + well differentiated PC & $14(11 \%)$ & $4(5 \%)$ & $10(8 \%)$ & $2(2 \%)$ \\
\hline Well differentiated PC & $15(11 \%)$ & $5(6 \%)$ & $20(15 \%)$ & $5(6 \%)$ \\
\hline Benign prostate + poorly differentiated PC & $2(2 \%)$ & $1(1 \%)$ & $2(2 \%)$ & 0 \\
\hline Well differentiated PC + poorly differentiated PC & $1(1 \%)$ & 0 & $1(1 \%)$ & 0 \\
\hline Poorly differentiated PC & $2(2 \%)$ & 0 & $1(1 \%)$ & 0 \\
\hline $\begin{array}{l}\text { Benign prostate + well differentiated PC + poorly } \\
\text { differentiated PC }\end{array}$ & $4(3 \%)$ & $3(3 \%)$ & $1(1 \%)$ & 1 (1\%) \\
\hline
\end{tabular}

by only $7 \%$ and $3 \%$ of UK and non-UK respondents, respectively, and as an external positive control by $4 \%$ and $1 \%$ of UK and non-UK respondents, respectively.

\section{DISCUSSION}

Sixty percent of the responding UK laboratories used a polyclonal anti-PSA antibody, whereas $40 \%$ used a monoclonal anti-PSA antibody. This bias may be a reflection of the relative insensitivity of monoclonal anti-PSA in the identification of poorly differentiated prostate cancer, the most common clinical setting for the use of prostate markers. ${ }^{45}$

Among the laboratories that measured PSAP, an equal number used monoclonal and polyclonal antibodies to PSAP. It is noteworthy that no systematic immunohistochemical comparison of the sensitivity of monoclonal and polyclonal anti-PSAP antibodies in high grade prostate cancer has been reported.

Although PSA immunohistochemistry was available in all laboratories using prostatic immunohistochemical markers, PSAP was used by only about half of them. However, two recent studies found PSAP to be more sensitive than PSA in high grade prostate cancer. ${ }^{56}$ Because the distinction of prostate cancer from other tumours, such as urothelial carcinoma, has important therapeutic implications and some cases of poorly differentiated prostate cancer may be negative for either PSA or PSAP, an immunohistochemical panel including both prostate markers is generally recommended. ${ }^{7}$ The need for a panel approach is also emphasised by the detection of PSAP immunoreactivity in a small number of non-prostatic tumours. ${ }^{8}$

\section{"Multiblocks containing benign prostate, well/moderately differentiated prostate cancer, and poorly differentiated prostate cancer may provide the ideal positive control for prostate specific antigen and prostate specific acid phosphatase immunohistochemistry"}

UKNEQAS-IHC quarterly reports indicate considerable variability in the results of prostate marker immunostaining from different laboratories. The variability may result from the use of different antigen retrieval methods, different clones or batches of anti-PSA and anti-PSAP antibodies, and different detection systems. It may be possible to overcome this variability through the use of standardised protocols and more stringent quality control.

Routine use of an appropriate (vide infra) external positive quality control capable of minimising the chance of a false negative result is highly recommended because many tumour biopsies may lack an adequate internal positive control component. It is noteworthy that almost one in 10 laboratories in the UK does not appear to include a positive external control for routine prostate marker analysis.

The selection of tissue for use as a positive control is also important because the use of too strongly positive tissue could mean that the lack of staining sensitivity is overlooked. It is known that PSA and PSAP expression is much higher in benign prostate glands and low grade prostate cancer than in high grade prostate cancer. However, most centres responding to our survey appear to use only benign prostate as an external positive control and risk overlooking false negative immunoreactivity.

Multiblocks containing tissues with varying sensitivities have been recommended for use in quality control. Multiblocks containing benign prostate, well/moderately differentiated prostate cancer, and poorly differentiated prostate cancer, as used by a single centre in the UK and another in continental Europe, may provide the ideal positive control for PSA and PSAP immunohistochemistry. Tissue microarrays, multiple small cores of different tissues embedded in a single block, would provide multiblock sections that could be immunostained on the same slide as the test tissue.' Another possible approach involves the use of solid matrix, cultured cells, expressing known and independently measured quantities of the target molecule. ${ }^{10}$

The weaker expression of PSA and PSAP in high grade prostate cancer also has implications for external quality assessment schemes. Most schemes like UKNEQAS-IHC have traditionally used sections of benign prostate to assess PSA and PSAP immunohistochemistry. However, sections of poorly differentiated prostate cancer would provide a more stringent evaluation of prostate marker immunostaining.

Linked to the issue of positive control tissues is the choice of tissue used to determine optimal immunostaining parameters, such as antibody dilution and incubation period. Currently, most laboratories appear to use sections of benign prostate and hence the assay sensitivity may be set too low. Only 7\% of laboratories in the UK included poorly differentiated prostate cancer in the tissues used to optimise PSA and PSAP immunohistochemistry. As for positive controls, multiblocks prepared by tissue microarray technique may be a solution.

The choice of tissues used to optimise immunostaining in previous studies of PSA and PSAP immunohistochemistry is generally unclear. It is likely that the antibody dilutions used in most or all of these studies may have been established using benign prostate and hence been too high for immunostaining high grade prostate cancer. Although in our experience polyclonal anti-PSA is very sensitive even in high grade prostate cancer, batch to batch variation could be an important problem, as with many polyclonal antibodies. If monoclonal antibodies to either PSA or PSAP, used at optimal dilutions established using poorly differentiated 


\section{Take home messages}

- There was a wide variation in the methods used for prostate specific antigen (PSA) and prostate specific acid phosphatase (PSAP) immunohistochemistry so that there is a need for standardisation and more stringent quality assurance

- A panel of antibodies to both PSA and PSAP should be used

- The widespread use of benign prostate tissue to determine optimal antibody dilutions and as an external positive control for PSA and PSAP immunostaining is of particular concern because it may result in a method that is not sufficiently sensitive to detect the reduced PSA and PSAP expression associated with high grade prostate cancer

- It is recommended that high grade prostate cancer is used for this purpose

prostate cancer, are found to be as sensitive as the corresponding polyclonal antibodies, then they may be the better antibody type because of their defined specificity and high quality assurance at the production and supply levels.

In conclusion, our study confirms that there is a wide variation in the immunohistochemical methods used in the diagnosis of prostate cancer. This variation probably reflects the fact that there has been no previous formal evaluation of the optimal techniques for detection of prostate markers. An evidence based methodology can only be identified by systematic comparisons of various primary antibodies and immunostaining methods, preferably by several different centres. In the absence of such a gold standard, immunohistochemistry in prostate cancer diagnosis should be used judiciously, preferably with a panel of markers and always after careful correlation with morphology. We also recommend using high grade prostate cancer to determine optimal antibody dilutions and as an external quality control in routine diagnostic practice, in addition to external quality assurance schemes.

\section{Authors' affiliations}

M Varma, B Jasani, University Hospital of Wales, Heath Park, Cardiff CF14 4XN, Wales, UK

D M Berney, St Bartholomew's Hospital, London ECIA 7BE, UK

A Rhodes, University of West of England, Bristol BS16 1QY, UK.

Previously manager of UKNEQAS-HC

\section{REFERENCES}

1 Wick MR, Mills SE. Consensual interpretive guidelines for diagnostic immunohistochemistry. Am J Surg Pathol 2001;25:1208-10.

2 Seidel T, Balaton AJ, Battifora H. Interpretation and quantification of immunostains. Am J Surg Pathol 2001;25:1204-7.

3 Oliai BR, Kahane H, Epstein Jl. A clinicopathologic analysis of urothelial carcinomas diagnosed on prostate needle biopsy. Am I Surg Pathol 2001:25:794-801.

4 Varma M, Morgan M, Jasani MB, et al. Polyclonal anti-PSA is more sensitive but less specific than monoclonal anti-PSA: implications for diagnostic prostatic pathology. Am J Clin Pathol 2002;1 18:202-7.

5 Goldstein NS. Immunophenotypic characterization of 225 prostate adenocarcinomas with intermediate or high Gleason scores. Am J Clin Pathol 2002;117:471-7.

6 Mhawech P, Uchida T, Pelte M. Immunohistochemical profile of high-grade urothelial carcinoma and prostate adenocarcinoma. Hum Pathol 2002:33:1136-40.

7 Epstein JI. PSA and PAP as immunohistochemical markers in prostate cancer. Urol Clin North Am 1993;20:757-69.

8 Bostwick DG. Neoplasms of the prostate. In: Bostwick DG, Eble JN, eds. Urologic surgical pathology, 1st ed. St Louis USA: Mosby, 1997:344-421.

9 von Wasielewski R, Mengel M, Wiese B, et al. Tissue array technology for testing interlaboratory and interobserver reproducibility of immunohistochemical estrogen receptor analysis in a large multicenter trial. Am J Clin Pathol 2002;118:675-82.

10 Riera J, Simpson JF, Tamayo R, et al. Use of cultured cells as a control for quantitative immunocytochemical analysis of estrogen receptor in breast cancer. The Quickgel method. Am J Clin Pathol 1999;111:329-35. 